\title{
Volatility Dynamics of the Greater China Stock Markets: A Multivariate Asymmetric Approach
}

\author{
Kin-Yip Ho ${ }^{1}$ \\ ${ }^{1}$ Department of Economics Cornell University Ithaca NY 14853-7601 USA
}

\begin{abstract}
This paper examines the volatility dynamics of the greater China stock markets (Shanghai A- and Bshares, Shenzhen A- and B-shares, Taiwan, and Hong Kong) by employing a multivariate (tetravariate) framework that incorporates the features of asymmetries, persistence, and time-varying correlations, which are typically observed in stock markets of developed economies. Specifically, we introduce two new multivariate GARCH models that do not nest each other: the Varying-Correlations (VC)fractionally integrated asymmetric power ARCH (VCFIAPARCH) and the VC-fractionally integrated asymmetric GARCH (VC-FIAGARCH) models. Our results indicate that, unlike the Shenzhen and Shanghai A-shares, Hong Kong, and Taiwan markets, both the B-share markets do not exhibit significant asymmetric volatility ("leverage effect"). Furthermore, return volatility in the A-share market is substantially higher than the B-share market before April 1997, but this result is reversed after that. Also, there is strong evidence of volatility persistence in all the markets, and this finding is robust to changes in model specification. It also appears possible that all the greater China stock markets share a common degree of persistence in volatility. Our examination of the correlation dynamics of these markets indicate that the Shenzhen and Shanghai stock exchanges are highly positively correlated with each other, with the strength of correlation increasing after the late nineties. Their correlations with the Hong Kong and Taiwanese markets, however, are much weaker and do not display any clear trends.
\end{abstract}

Keywords: Multivariate GARCH; Time-varying correlations; Volatility asymmetry and persistence

\section{Introduction}

The interrelationships among the financial markets in the greater China area, which comprises mainland China, Taiwan, and Hong Kong SAR, is a noteworthy issue of economic research, mainly because the financial markets in this region have grown rapidly. In particular, among all the members of the World Federation of Exchanges, the Hong Kong Stock Exchange ranks 9th in the world by market capitalization in 2004. In the case of the Taiwan Stock Exchange, a major liberalization took place in May 1988 and the number of listed companies increased rapidly from 199 in 1990 to 669 in 2003 . As for the mainland Chinese stock markets, they have also entered a new stage of development, as manifested by the exponential expansion of market capitalization. By the end of 2004, the 837 companies listed on the Shanghai Stock Exchange had a total market capitalization of US\$ 322.477 billion. On the other hand, by 2005, market capitalization in the Shenzhen stock market amounted to US\$ 122 billion.

In view of the growing importance of the greater China stock markets, it would be interesting to model and analyze the volatility dynamics of these markets. This paper proposes a unified approach of doing so by adopting a multivariate framework that simultaneously incorporates asymmetries, persistence, and timevarying (dynamic) correlations. Specifically, we introduce two new multivariate generalized autoregressive conditional heteroscedasticity (GARCH) models that do not nest each other: the Varying-Correlations (VC)-fractionally integrated asymmetric power ARCH (VC-FIAPARCH) and the VC-fractionally integrated asymmetric GARCH (VCFIAGARCH) models. The models that we propose have these advantages:

- They nest other popular GARCH models that exist in the literature (see Ding, Granger, and Engle (1993) and Sentana (1995) for details);

- They guarantee that the variance-covariance matrix is positive-definite once parameter estimates are obtained;

- They are relatively parsimonious compared with other multivariate models found in the literature, such as the BEKK model of Engle and Kroner (1995);

- All equations are estimated simultaneously instead of using a multi-step procedure. This 
is deemed more efficient than Engle's (2002)

Dynamic Correlation (DC) approach.

The rest of this paper is organized as follows. Section 2 outlines the econometric methodology used in this paper. The next section then discusses the data sets employed in this paper and analyzes the estimation results.

\section{Econometric Methodology}

We outline the basic features of the following multivariate GARCH models in this section: the VCQGARCH, the VC-APARCH, the VC-FIAGARCH and the VC-FIAPARCH.

\subsection{Time-Varying Correlations}

First, we briefly describe the VC-GARCH model, the details of which are available in Tse and Tsui (2002):

- Conditional Mean $y_{i t}$ :

$y_{i t}=\mu_{i t}\left(\xi_{i}\right)+\varepsilon_{i t}, \quad i=1,2, \ldots, k$

where $\mu_{i t}\left(\xi_{i}\right)$ is the conditional mean function, $\varepsilon_{\mathrm{it}}$ is the random disturbance term, and

$\left(\varepsilon_{1 t}, \varepsilon_{2 t}, \varepsilon_{3 t}, \ldots, \varepsilon_{k t}\right)^{\prime} \mid \Phi_{t-1} \sim\left(O, H_{t}\right)$

$\Phi_{\mathrm{t}-1}$ is the $\sigma$-algebra generated by all the available information up to time (t-1).

- Conditional Variance $h_{i i t}$ :

$\varepsilon_{i t}=\sqrt{h_{i i t}} e_{i t}, \quad$ where $e_{i t} \sim N(0,1)$

$h_{i i t}=\eta_{i}+\alpha_{i} \varepsilon_{i t-1}^{2}+\beta_{i} h_{i i t-1}, \quad i=1, \ldots, k$

- Conditional Correlation Matrix $\Gamma_{t}=\left\{\rho_{i j t}\right\}$ :

$\boldsymbol{\Gamma}_{t}=\left(1-\pi_{1}-\pi_{2}\right) \boldsymbol{\Gamma}+\pi_{1} \boldsymbol{\Gamma}_{t-1}+\pi_{2} \Psi_{t-1}$

where $\rho_{i j t}=\frac{h_{i j t}}{\sqrt{h_{i i t} h_{j j t}}}, \mathrm{~h}_{\mathrm{ijt}}$ is the conditional covariance between variables $\mathrm{i}$ and $\mathrm{j}, \Gamma=\left\{\rho_{i j}\right\}$ is a time-invariant ( $\mathrm{k} \times \mathrm{k}$ ) positive-definite correlation matrix, $\pi_{1}$ and $\pi_{2}$ are assumed to be nonnegative and sum up to less than 1 , and $\Psi_{\mathrm{t}}$ is a function of the standardised residuals $e_{i t}$. Denoting $\Psi_{\mathrm{t}}=\left\{\psi_{i j t}\right\}$, the elements of $\Psi_{\mathrm{t}-1}$ are specified as

$\psi_{i j, t-1}=\frac{\sum_{a=1}^{M} e_{i, t-a} e_{j, t-a}}{\sqrt{\left(\sum_{a=1}^{M} e_{i, t-a}^{2}\right)\left(\sum_{a=1}^{M} e_{j, t-a}^{2}\right)}}, \quad 1 \leq i<j \leq k$

where $\mathrm{M}$ is set equal to $\mathrm{k}$, in accordance with Tse and Tsui (2002).

- Log-likelihood Function (assuming normality conditional on $\Phi_{\mathrm{t}-1}$ and ignoring the constant term): $l_{t}(\theta)=-\frac{1}{2} \log \left|H_{t}\right|-\frac{1}{2}\left(\varepsilon_{1 t}, \varepsilon_{2 t}, \varepsilon_{3 t}, \ldots, \varepsilon_{k t}\right) H_{t}^{-1}\left(\varepsilon_{1 t}, \varepsilon_{2 t}, \varepsilon_{3 t}, \ldots, \varepsilon_{k t}\right)^{\prime}(7)$

where

$\theta=\left(\xi_{1}, \xi_{2}, \ldots, \xi_{k}, \eta_{1}, \eta_{2}, \ldots, \eta_{k}, \alpha_{1}, \ldots, \alpha_{k}, \beta_{1}, \ldots, \beta_{k}, \rho_{i j}, \pi_{1}, \pi_{2}\right)$

and $\varepsilon_{\text {it }}$ are the random disturbance terms obtained from equation (1).

Alternatively, $l_{t}(\theta)$ can be rewritten as

$l_{t}(\theta)=-\frac{1}{2} \log \left|D_{t} \Gamma_{t} D_{t}\right|-\frac{1}{2}\left(\varepsilon_{1 t}, \varepsilon_{2 t}, \varepsilon_{3 t}, \ldots, \varepsilon_{k t}\right) D_{t}^{-1} \Gamma_{t}^{-1} D_{t}^{-1}\left(\varepsilon_{1 t}, \varepsilon_{2 t}, \varepsilon_{3 t}, \ldots, \varepsilon_{k t}\right)^{\prime}$

if we define the conditional variance-covariance matrix $\mathrm{H}_{\mathrm{t}}$ as $H_{t}=\left\{h_{i j t}\right\} \equiv D_{t} \Gamma_{\mathrm{t}} D_{t}$, where $D_{t}=\operatorname{diag}\left\{\sqrt{h_{i i t}}\right\}$, and $\Gamma_{t}=\left\{\rho_{i j t}\right\}$. By this formulation, $\left(\varepsilon_{1 t}, \varepsilon_{2 t}, \varepsilon_{3 t}, \ldots, \varepsilon_{k t}\right) D_{t}^{-1}$ represents the standardized residuals $\left(e_{1 t}, e_{2 t} \ldots e_{k t}\right)$. As noted by Tse and Tsui (2002), Bollerslev's (1990) constant-correlations GARCH (CC-GARCH) model is nested within the VC-GARCH model under the restrictions $\pi_{1}=\pi_{2}=0$. Thus, the likelihood ratio test can be readily applied to compare the performance of these two models.

\subsection{Asymmetric Volatility}

Next, we outline the VC-QGARCH model. ${ }^{1}$ Essentially, only the conditional variance equation (4) changes whilst everything else remains the same:

$h_{t}=\eta-\gamma \varepsilon_{t-1}+\alpha \varepsilon_{t-1}^{2}+\beta h_{t-1}$

where $\gamma$ is the asymmetric coefficient. Sentana's (1995) QGARCH $(1,1)$ model is related to Engle's (1990) AGARCH $(1,1)$ model when we rearrange the terms in equation (9):

$$
h_{t}=\eta-\frac{\gamma^{2}}{4 \alpha}+\alpha\left(\varepsilon_{t-1}-\frac{\gamma}{2 \alpha}\right)^{2}+\beta h_{t-1}
$$

If we redefine $\eta^{*} \equiv \eta-\frac{\gamma^{2}}{4 \alpha}$ and $\gamma^{*} \equiv \frac{\gamma}{2 \alpha}$, then the $\operatorname{AGARCH}(1,1)$ model is obtained:

$$
h_{t}=\eta^{*}+\alpha\left(\varepsilon_{t-1}-\gamma^{*}\right)^{2}+\beta h_{t-1}
$$

Similarly, to specify the VC-APARCH model, we modify equation (4) as follows:

$$
h_{t}^{\delta / 2}=\eta+\alpha\left(\left|\varepsilon_{t-1}\right|-\gamma \varepsilon_{t-1}\right)^{\delta}+\beta h_{t-1}^{\delta / 2}
$$

where $\gamma$ is the asymmetric coefficient and $h_{t}^{\delta / 2}$ is the power transformation of the conditional variance.

\subsection{Long-memory Dynamics}

To model long-memory dynamics, we employ the fractionally integrated approach (Baillie, Bollerslev, and Mikkelsen (1996)). Specifically, we rearrange equation (4) and factorize the lag polynomial $1-\beta L-\alpha L$ as $(1-L)^{d}(1-\phi L)$ :

$(1-L)^{d}(1-\phi L) \varepsilon_{t}^{2}=\eta+[1-\beta L] v_{t}$

\footnotetext{
${ }^{1}$ To simplify the notation, the subscript $\mathrm{i}$ is omitted.
} 
where $v_{t}=\varepsilon_{t}^{2}-h_{t}$. By manipulating equation (13) algebraically, we obtain the FIGARCH( $(1, \mathrm{~d}, 1)$ model:

$h_{t}=\frac{\eta}{1-\beta}+\lambda(L) \varepsilon_{t}^{2}$

where $\lambda(L)=\sum_{a=1}^{\infty} \lambda_{a} L^{a}=1-(1-\beta L)^{-1}(1-\phi L)(1-L)^{d}$. To incorporate asymmetries into equation (13), we apply the fractional differencing operator $(1-L)^{d}$ to the AGARCH and APARCH models in equations (11) and (12) respectively:

- $\quad$ FIAGARCH( $(1, \mathrm{~d}, 1)$ :

$h_{t}=\frac{\eta^{*}}{1-\beta}+\lambda(L)\left(\varepsilon_{t}-\gamma^{*}\right)^{2}$

- $\quad$ FIAPARCH( $(1, \mathrm{~d}, 1)$ :

$h_{t}^{\delta / 2}=\frac{\eta}{1-\beta}+\lambda(L)\left(\left|\varepsilon_{t}\right|-\gamma \varepsilon_{t}\right)^{\delta}$

By combining the FIGARCH, FIAGARCH, and FIAPARCH models with the varying-correlation structure described in Section 2.1, we obtain the VCFIGARCH, VC-FIAGARCH, and VC-FIAPARCH models. The parameters of all the models are estimated using Bollerslev and Wooldridge's (1992) quasi-maximum likelihood estimation (QMLE) approach. To compute the response coefficients in $\lambda(L)$, we adopt the following infinite recursions given in Bollerslev and Mikkelsen (1996):

$$
\begin{aligned}
& \lambda_{1}=\phi-\beta+d, \\
& \lambda_{b}=\beta \lambda_{b-1}+[(b-1-d) / b-\phi] \zeta_{b-1}, \quad b=2, \ldots, \infty
\end{aligned}
$$$$
\text { where } \zeta_{b}=\zeta_{b-1}(b-1-d) / b \text {, with } \zeta_{1}=d
$$

\section{Data and Estimation Results}

The following data sets are used in this paper: the Shanghai Composite, A-share, and B-share Indices (SHSCOMP, SHSASHR, SHSBSHR), the Shenzhen Composite, A-share and B-share Indices (SHZCOMP, SHZASHR, SHZBSHR), the Taiwan Weighted Index (TAIWGHT), and the Hang Seng Index (HNGKNGI). Specifically, for the SHSCOMP, SHZCOMP, TAIWGHT, and HNGKNGI indices, the data sets comprise 3225 daily price observations from May 22 1992 to September 30 2004. As for the A-share and Bshare indices, instead of May 22 1992, the start date of our analysis is October 5 1992, because this is the very first day of availability for the Shenzhen A-share and B-share indices. All data sets are obtained from DataStream International.

Our main findings are as follows:

- Asymmetric volatility ("leverage effect") is detected for most markets and this is robust across various model specifications; for the
Shanghai and Shenzhen markets, only the Bshare indices do not exhibit significant asymmetric effects.

- Volatility persistence is detected in all markets, as measured by the significant fractional differencing parameter $\mathrm{d}$.

- All the markets might share a common degree of volatility persistence (fractional integration).

- Low correlation coefficients of the mainland Chinese markets with those of Hong Kong and Taiwan suggest that Shanghai and Shenzhen are relatively insulated from the rest of the region.

- The correlation between the Shanghai and Shenzhen markets are relatively strong but not perfectly positive.

- Time-varying correlations exist among the markets. In particular, there is evidence that correlations are strengthening over time.

- There is substantial volatility disparity between the A-share and B-share markets. Before April 1997, the volatility of the Ashare indices is higher than that of their Bshare counterparts, but after that, the B-share markets exhibit greater fluctuations.

\section{References}

[1] R. Baillie, T. Bollerslev, and H. Mikkelsen, "Fractionally Integrated Generalized Autoregressive Conditional Heteroskedasticity," Journal of Econometrics 74, pp. 3-30, 1996.

[2] T. Bollerslev, and R. Engle, "Common Persistence in Conditional Variances," Econometrica 61, pp. 166-187, 1993.

[3] T. Bollerslev, and H. Mikkelsen, "Modeling and Pricing Long Memory in Stock Market Volatility," Journal of Econometrics 73, pp. 151-184, 1996.

[4] T. Bollerslev, and J. Wooldridge, "QuasiMaximum Likelihood Estimation and Inference in Dynamic Models with Time-Varying Covariances," Econometric Reviews 11, pp. 143-172, 1992.

[5] Z. Ding, R. Engle, and C. Granger, "A Longmemory Property of Stock Market Returns and a New Model," Journal of Empirical Finance 1, pp. 107-131, 1993.

[6] R. Engle, "Dynamic Conditional Correlation: A Simple Class of Multivariate Generalized Autoregressive Conditional Heteroskedasticity 
Models," Journal of Business and Economic Statistics 20.3, pp. 339-350, 2002.

[7] R. Engle, and K. Kroner, "Multivariate Simultaneous Generalized ARCH," Econometric Theory 11, pp. 122-150, 1995.

[8] E. Sentana, "Quadratic ARCH Models," Review of Economic Studies 62, pp. 639-661, 1995.

[9] Shanghai Stock Exchange, "Shanghai Stock Exchange Fact Book 2004," 2005. http://www.sse.com.cn/en_us/cs/about/factbook/factbo ok_us2004.pdf

[10] Y. Tse, and A Tsui, "A Multivariate Generalized Autoregressive Conditional Heteroskedasticity Model with Time-varying Correlations," Journal of Business and Economic Statistics 20, pp. 351-362, 2002.

\begin{tabular}{|c|c|c|c|c|}
\hline Model & $\begin{array}{l}\text { VC- } \\
\text { FIAGARCH }\end{array}$ & $\begin{array}{l}\text { VC- } \\
\text { FIAPARCH }\end{array}$ & $\begin{array}{l}\text { VC- } \\
\text { QGARCH }\end{array}$ & $\begin{array}{l}\text { VC- } \\
\text { APARCH }\end{array}$ \\
\hline \multicolumn{5}{|c|}{ Group 1 (Composite Indices) } \\
\hline TAIWGHT & $\begin{array}{l}0.9832 \\
(0.1787)\end{array}$ & $\begin{array}{l}0.3428 \\
(0.0783)\end{array}$ & $\begin{array}{l}0.1557 \\
(0.0431)\end{array}$ & $\begin{array}{l}0.3214 \\
(0.0790)\end{array}$ \\
\hline HNGKNGI & $\begin{array}{l}0.6740 \\
(0.1948)\end{array}$ & $\begin{array}{l}0.3932 \\
(0.1112)\end{array}$ & $\begin{array}{l}0.0891 \\
(0.0260)\end{array}$ & $\begin{array}{l}0.3703 \\
(0.0791)\end{array}$ \\
\hline CHSCOMP & $\begin{array}{l}0.2427 \\
(0.1272)\end{array}$ & $\begin{array}{l}0.0980 \\
(0.0517)\end{array}$ & $\begin{array}{l}0.0379 \\
(0.0162)\end{array}$ & $\begin{array}{l}0.1586 \\
(0.0548)\end{array}$ \\
\hline CHZCOMP & $\begin{array}{l}0.3314 \\
(0.1077)\end{array}$ & $\begin{array}{l}0.1313 \\
(0.0468)\end{array}$ & $\begin{array}{l}0.0432 \\
(0.0165)\end{array}$ & $\begin{array}{l}0.1689 \\
(0.0515)\end{array}$ \\
\hline \multicolumn{5}{|c|}{ Group 2 (A-share Indices) } \\
\hline TAIWGHT & $\begin{array}{l}0.9775 \\
(0.1759)\end{array}$ & $\begin{array}{l}0.3327 \\
(0.0799)\end{array}$ & $\begin{array}{l}0.1518 \\
(0.0430)\end{array}$ & $\begin{array}{l}0.3073 \\
(0.0766)\end{array}$ \\
\hline HNGKNGI & $\begin{array}{l}0.6286 \\
(0.1731) \\
\end{array}$ & $\begin{array}{l}0.3927 \\
(0.1079) \\
\end{array}$ & $\begin{array}{l}0.0865 \\
(0.0267)\end{array}$ & $\begin{array}{l}0.3687 \\
(0.0813)\end{array}$ \\
\hline CHSASHR & $\begin{array}{l}0.1697 \\
(0.1254) \\
\end{array}$ & $\begin{array}{l}0.0480 \\
(0.0535) \\
\end{array}$ & $\begin{array}{l}0.0317 \\
(0.0158) \\
\end{array}$ & $\begin{array}{l}0.1294 \\
(0.0569) \\
\end{array}$ \\
\hline CHZASHR & $\begin{array}{l}0.2714 \\
(0.1066) \\
\end{array}$ & $\begin{array}{l}0.0797 \\
(0.0461) \\
\end{array}$ & $\begin{array}{l}0.0390 \\
(0.0156) \\
\end{array}$ & $\begin{array}{l}0.1426 \\
(0.0537) \\
\end{array}$ \\
\hline \multicolumn{5}{|c|}{ Group 3 (B-share Indices) } \\
\hline TAIWGHT & $\begin{array}{l}0.9764 \\
(0.1912) \\
\end{array}$ & $\begin{array}{l}0.3358 \\
(0.0819) \\
\end{array}$ & $\begin{array}{l}0.1476 \\
(0.0419) \\
\end{array}$ & $\begin{array}{l}0.3050 \\
(0.0776) \\
\end{array}$ \\
\hline HNGKNGI & $\begin{array}{l}0.5805 \\
(0.4378)\end{array}$ & $\begin{array}{l}0.3562 \\
(0.1081)\end{array}$ & $\begin{array}{l}0.0736 \\
(0.0237)\end{array}$ & $\begin{array}{l}0.3436 \\
(0.0785)\end{array}$ \\
\hline CHSBSHR & $\begin{array}{l}-0.0013 \\
(0.1234) \\
\end{array}$ & $\begin{array}{l}0.0112 \\
(0.0453) \\
\end{array}$ & $\begin{array}{l}-0.0108 \\
(0.0408) \\
\end{array}$ & $\begin{array}{l}-0.0048 \\
(0.0479) \\
\end{array}$ \\
\hline CHZBSHR & $\begin{array}{l}-0.1279 \\
(0.1308)\end{array}$ & $\begin{array}{l}-0.0465 \\
(0.0442)\end{array}$ & $\begin{array}{l}-0.0621 \\
(0.0489)\end{array}$ & $\begin{array}{l}-0.0422 \\
(0.0412)\end{array}$ \\
\hline
\end{tabular}

Table 1: Estimated values of the asymmetry parameter $\gamma$. The figures in brackets are the BollerslevWooldridge (1992) heteroskedastic-consistent standard errors.

\begin{tabular}{|l|l|l|l|}
\hline Model & $\begin{array}{l}\text { VC- } \\
\text { FIGARCH }\end{array}$ & $\begin{array}{l}\text { VC- } \\
\text { FIAGARCH }\end{array}$ & $\begin{array}{l}\text { VC- } \\
\text { FIAPARCH }\end{array}$ \\
\hline Group 1 (Composite Indices) \\
\hline TAIWGHT & $\begin{array}{l}0.3000 \\
(0.0512)\end{array}$ & $\begin{array}{l}0.2506 \\
(0.0432)\end{array}$ & $\begin{array}{l}0.2723 \\
(0.0502)\end{array}$ \\
\hline HNGKNGI & 0.3963 & 0.2957 & 0.3060 \\
\hline & $(0.1048)$ & $(0.0703)$ & $(0.1191)$ \\
\hline
\end{tabular}

\begin{tabular}{|c|c|c|c|}
\hline Model & $\begin{array}{l}\text { VC- } \\
\text { FIGARCH }\end{array}$ & $\begin{array}{l}\text { VC- } \\
\text { FIAGARCH }\end{array}$ & $\begin{array}{l}\text { VC- } \\
\text { FIAPARCH }\end{array}$ \\
\hline CHSCOMP & $\begin{array}{l}0.3559 \\
(0.0430)\end{array}$ & $\begin{array}{l}0.3456 \\
(0.0419)\end{array}$ & $\begin{array}{l}0.3504 \\
(0.0404)\end{array}$ \\
\hline CHZCOMP & $\begin{array}{l}0.3684 \\
(0.0391)\end{array}$ & $\begin{array}{l}0.3515 \\
(0.0348)\end{array}$ & $\begin{array}{l}0.3566 \\
(0.0341)\end{array}$ \\
\hline \multicolumn{4}{|c|}{ Group 2 (A-share Indices) } \\
\hline TAIWGHT & $\begin{array}{l}0.3015 \\
(0.0537) \\
\end{array}$ & $\begin{array}{l}0.2544 \\
(0.0437) \\
\end{array}$ & $\begin{array}{l}0.2692 \\
(0.0498) \\
\end{array}$ \\
\hline HNGKNGI & $\begin{array}{l}0.4166 \\
(0.1145)\end{array}$ & $\begin{array}{l}0.3146 \\
(0.0770)\end{array}$ & $\begin{array}{l}0.3708 \\
(0.1284)\end{array}$ \\
\hline CHSASHR & $\begin{array}{l}0.3731 \\
(0.0405)\end{array}$ & $\begin{array}{l}0.3642 \\
(0.0396)\end{array}$ & $\begin{array}{l}0.3686 \\
(0.0398) \\
\end{array}$ \\
\hline CHZASHR & $\begin{array}{l}0.3726 \\
(0.0380) \\
\end{array}$ & $\begin{array}{l}0.3554 \\
(0.0338) \\
\end{array}$ & $\begin{array}{l}0.3633 \\
(0.0339)\end{array}$ \\
\hline \multicolumn{4}{|c|}{ Group 3 (B-share Indices) } \\
\hline TAIWGHT & $\begin{array}{l}0.2928 \\
(0.0504) \\
\end{array}$ & $\begin{array}{l}0.2454 \\
(0.0490) \\
\end{array}$ & $\begin{array}{l}0.2630 \\
(0.0480)\end{array}$ \\
\hline HNGKNGI & $\begin{array}{l}0.4167 \\
(0.1338)\end{array}$ & $\begin{array}{l}0.3084 \\
(0.0870)\end{array}$ & $\begin{array}{l}0.4031 \\
(0.1682)\end{array}$ \\
\hline CHSBSHR & $\begin{array}{l}0.3487 \\
(0.0583)\end{array}$ & $\begin{array}{l}0.3466 \\
(0.0584)\end{array}$ & $\begin{array}{l}0.3698 \\
(0.0654)\end{array}$ \\
\hline CHZBSHR & $\begin{array}{l}0.2921 \\
(0.0397)\end{array}$ & $\begin{array}{l}0.2885 \\
(0.0437) \\
\end{array}$ & $\begin{array}{l}0.2654 \\
(0.0333)\end{array}$ \\
\hline
\end{tabular}

Table 2: Fractional differencing parameter d. The figures in brackets are the Bollerslev-Wooldridge (1992) heteroskedastic-consistent standard errors.

\begin{tabular}{|c|c|c|c|}
\hline Model & $\begin{array}{l}\text { VC- } \\
\text { FIGARCH }\end{array}$ & $\begin{array}{l}\text { VC- } \\
\text { FIAGARCH }\end{array}$ & $\begin{array}{l}\text { VC- } \\
\text { FIAPARCH }\end{array}$ \\
\hline \multicolumn{4}{|c|}{ Group 1 (Composite Indices) } \\
\hline $\begin{array}{l}\text { TAIWGHT- } \\
\text { HNGKNGI }\end{array}$ & $\begin{array}{l}0.3965 \\
(0.0745)\end{array}$ & $\begin{array}{l}0.4085 \\
(0.0779)\end{array}$ & $\begin{array}{l}0.4031 \\
(0.0807)\end{array}$ \\
\hline $\begin{array}{l}\text { TAIWGHT- } \\
\text { CHSCOMP }\end{array}$ & $\begin{array}{l}0.0685 \\
(0.0650)\end{array}$ & $\begin{array}{l}0.0604 \\
(0.0667)\end{array}$ & $\begin{array}{l}0.0606 \\
(0.0737)\end{array}$ \\
\hline $\begin{array}{l}\text { TAIWGHT- } \\
\text { CHZCOMP }\end{array}$ & $\begin{array}{l}0.0701 \\
(0.0655)\end{array}$ & $\begin{array}{l}0.0607 \\
(0.0669)\end{array}$ & $\begin{array}{l}0.0604 \\
(0.0716)\end{array}$ \\
\hline $\begin{array}{l}\text { HNGKNGI- } \\
\text { CHSCOMP }\end{array}$ & $\begin{array}{l}0.0134 \\
(0.0421)\end{array}$ & $\begin{array}{l}0.0026 \\
(0.0337)\end{array}$ & $\begin{array}{l}-0.0029 \\
(0.1108)\end{array}$ \\
\hline $\begin{array}{l}\text { HNGKNGI- } \\
\text { CHZCOMP }\end{array}$ & $\begin{array}{l}0.0313 \\
(0.0404)\end{array}$ & $\begin{array}{l}0.0183 \\
(0.0342)\end{array}$ & $\begin{array}{l}0.0109 \\
(0.0933)\end{array}$ \\
\hline $\begin{array}{l}\text { CHSCOMP- } \\
\text { CHZCOMP }\end{array}$ & $\begin{array}{l}0.9872 \\
(0.0066)\end{array}$ & $\begin{array}{l}0.9870 \\
(0.0068)\end{array}$ & $\begin{array}{l}0.9880 \\
(0.0068)\end{array}$ \\
\hline$\pi_{1}$ & $\begin{array}{l}0.9678 \\
(0.0052)\end{array}$ & $\begin{array}{l}0.9685 \\
(0.0052)\end{array}$ & $\begin{array}{l}0.9696 \\
(0.0053)\end{array}$ \\
\hline$\pi_{2}$ & $\begin{array}{l}0.0244 \\
(0.0036)\end{array}$ & $\begin{array}{l}0.0242 \\
(0.0036)\end{array}$ & $\begin{array}{l}0.0234 \\
(0.0035)\end{array}$ \\
\hline
\end{tabular}

Table 3: Conditional Correlation Matrix $\Gamma_{\mathrm{t}}$. The figures in brackets are the Bollerslev-Wooldridge (1992) heteroskedastic-consistent standard errors. 